\title{
High prevalence of Insomnia during COVID-19 pandemic
}

\begin{abstract}
The authors review the evidence suggesting greater prevalence of insomnia during the COVID-19 global pandemic. Given the evidence of the role of sleep plays in immune response, sleep or lack thereof may be a modifiable risk factor during the current pandemic.
\end{abstract}

Keywords: COVID 19, sleep, insomnia, stress
Volume 4 Issue 2 - 2020

\author{
Kawish Garg,' Harsh Goel,'2 Shefali D Gupta ${ }^{3}$ \\ 'Geisinger Holy Spirit Sleep Medicine, USA \\ ${ }^{2}$ St. Luke's University Hospital, Department of Internal Medicine, \\ USA \\ ${ }^{3}$ Geisinger Holy Spirit Hospital, Department of Medicine, USA
}

\author{
Correspondence: Kawish Garg MD, Geisinger Holy Spirit \\ Sleep Medicine, 550 N I2th St, Camp Hill, PA, USA, Tel \\ 7019361980,Email Kgarg@geisinger.edu \\ Received:September 16, 2020 | Published:September 30 \\ 2020
}

Abbreviations: PHEIC, public health emergency of international concern; WHO, World health organization; SARS, severe acute respiratory syndrome

\section{Introduction}

Coronavirus disease 2019, also known as COVID-19 first emerged in Wuhan, China in December 2019 and since then, has rapidly spread globally. It was declared as public Health Emergency of international concern (PHEIC) by the World Health Organization on $30^{\text {th }}$ Jan 2020, and a pandemic on $11^{\text {th }}$ March 2020. ${ }^{2}$ As of time of writing this report, there are $>20$ million confirmed cases of COVID-19, and $>750,000$ confirmed deaths globally reported by WHO. As per CDC USA data reported on August 18, 2020, there are 5,422,242 cases reported in United States with 169,870 deaths. It has long been known that incidence of mental health problems including depression, anxiety, posttraumatic stress, and sleep disturbances rises during disease pandemics/epidemics. ${ }^{3}$ Given the uniquely high impact of the current pandemic, it should hardly be surprising if a similar, or indeed worse, pattern be observed during the COVID-19 pandemic. Moreover, these mental health problems likely have long-lasting affect, beyond the duration of the pandemic. ${ }^{4}$ Beyond the obvious cost in terms of mortality and morbidity due to the infection itself, the unprecedented spread of the pandemic has caused immense socioeconomic strain due to mandatory lockdowns across the developed and the developing world. For example, quarantine by itself is an independent risk factor for worsening emotional disturbance, depression, stress, low mood, irritability and anger, poor sleep, and posttraumatic stress symptoms. ${ }^{5}$ Various stressors during or post quarantine include fear of infection, frustration, and boredom, in adequate supplies, inadequate information, stigma and worry about finances. ${ }^{5}$

Among the myriad psychological complications of a global pandemic, sleep disturbances are among the more common, and indeed more easily quantifiable. ${ }^{6}$ Sleep is critical to physical and psychological well-being, ${ }^{7}$ and may even improve resilience and immunity to fight infections, not to mention myriad effects of sleep deprivation on metabolic, autonomic, and inflammatory processes. ${ }^{8}$ We do not have much epidemiological data regarding prevalence of insomnia during and after pandemics, with one study reporting a $\approx 12-19 \%$ incidence of sleep complaints during the 2003 severe acute respiratory syndrome (SARS) pandemic, incidentally also caused by a coronavirus. ${ }^{6}$ Many recent reports have attempted to assess the impact of the COVID-19 pandemic on incidence of sleep disorders. This review aims to summarize the evidence of the magnitude of the problem of impaired sleep during the current pandemic.

\section{Discussion}

As per published literature, prevalence of insomnia ranged from $15.7 \%$ to as high as $37.6 \%$. This prevalence is relatively higher than reported insomnia in epidemiological studies in general population. There is quite a bit of heterogeneity in insomnia epidemiological data due to different measuring instruments used by studies, prevalence can vary from $6 \%$ if using DSM-IV diagnostic criteria for insomnia, to $36 \%$ if using just one or more symptoms of insomnia..$^{9,10}$ We do not know much about cause and effect relationship between COVID 19 and insomnia or even with other pandemics before, there is not much data available. One plausible hypothesis could be stress induced activation of both hypothalamic-pituitary-adrenocortical axis and sympathetic-adrenal-medullary system, hence increased secretion of cortisol and catecholamines-all lead to hyper arousal state hence insomnia, it needs to be explored further though. ${ }^{11,12}$ Other factors could be circadian misalignment, irregular sleep wake schedule, increased screen time, confinement to the houses, fear of uncertainty, increased use of substances or worsening of depression and anxiety etc. Most of the reviewed studies also tried to investigate the risk factors for development of insomnia related to COVID-19 pandemic and identified many similarities. Overall prevalence of insomnia was found to be higher in healthcare workers, females, urban citizens, increased use of internet, previous history of medical and psychological problems, lower income, relatively younger age $<40$, decrease in physical activity, lower education etc. Risk of insomnia was also found to be higher in family members or friends or relatives of patient with COVID-19 or healthcare workers.

Except one study, ${ }^{13}$ all studies reported females to have higher prevalence of Insomnia, which was consistent with the results from the previous insomnia epidemiological studies. Use of internet was also observed to be one of the risk factor for developing insomnia 
in these studies, we don't know much how internet would affect the prevalence of insomnia but it was suggested that it could be due to less social media exposure, less screen time and less worrying, provides important insight and to look further, how internet and social media might have influence on development of insomnia in general population. All of these studies were cross sectional in nature, and didn't have much information regarding subjects' previous psychological health except few, so difficult to develop causal relationship, and we cannot eliminate other confounding factors effectively which might have also played a role in worsening of insomnia. These studies were also performed in different geographical areas, at different times during pandemic, all of the studies used web-based surveys which can cause selection bias as only the subjects who were educated or had access to technology were included Table 1.

Table I Findings from studies below

\begin{tabular}{|c|c|c|c|c|}
\hline Authors & Country & Sample size & Scales & Insomnia prevalence \\
\hline Huang et al., ${ }^{14}$ & China & 7236 & PSQI (Cut off 7) & $18.20 \%$ \\
\hline Guo et al., ${ }^{15}$ & China & $244 I$ & PSQI (Cut off 7) & $20 \%$ \\
\hline Fu et al., ${ }^{16}$ & China & 1242 & Athens Insomnia scale (Cut off 5) & $30 \%$ \\
\hline Voitsidis et al., ${ }^{17}$ & Greece & 2363 & Athens Insomnia scale (Cut off 6) & $37.60 \%$ \\
\hline Gualano et al., ${ }^{18}$ & Italy & 1515 & ISI (Cut off 8) & $33 \%$ \\
\hline Shi et al., ${ }^{13}$ & China & 56679 & ISI (Cut off 8) & $29.20 \%$ \\
\hline Kokou-Kpolou et al., ${ }^{19}$ & France & 556 & ISI (Cut off I5) & $19.10 \%$ \\
\hline Pieh et al., ${ }^{20}$ & Austria & 1005 & ISI (Cut off I5) & $15.70 \%$ \\
\hline Yu et al., ${ }^{21}$ & Hong Kong (China) & 1138 & ISI (Cut off I0) & $29.90 \%$ \\
\hline Lin et al., ${ }^{22}$ & China & 5641 & ISI (Cut off I0) & $20.05 \%(20.6 \%$ based on cut off 8$)$ \\
\hline Li et al., ${ }^{23}$ & China & 3637 & ISI (Cut off 7) & $33.70 \%$ \\
\hline
\end{tabular}

- PSQI- Pittsburgh sleep quality index, 19 item self-reported questionnaire, with 5 additional questions from roommate or bed partner, maximum score 21 , score above 5 indicate poor sleep quality. It evaluates sleep among 7 domains including sleep disturbance, sleep onset latency, duration, daytime dysfunction, sleep efficiency, need of medications to sleep and overall sleep quality.

- Insomnia severity index is 7 item self-reported scales, 0 to 7 indicates no significant insomnia, 8 to I4-sub threshold insomnia, I5 to 21 -moderate and 22 to 28 indicates severe insomnia.

- Athens insomnia scale is 8 item self-reported questionnaires with score more than 6 indicate insomnia. 0 to $<4$-no clinically significant insomnia, 4 to 6-risk of developing insomnia.

\section{Conclusion}

All the published studies collectively suggest high prevalence of insomnia during COVID 19. Since sleep has a role in health of the immune response and psychological health, poor sleep may be a modifiable risk factor for decreasing COVID 19 related severe morbidity and mortality.

\section{Acknowledgments}

None.

\section{Conflicts of interest}

The authors declare that they have no conflicts of interest.

\section{Funding}

None.

\section{References}

1. WHO. Rolling updates on coronavirus disease (COVID-19). 2020.

2. WHO. Archived: WHO timeline-COVID-19. WHO. 2020.
3. Morganstein JC, Fullerton CS, Ursano RJ, et al. Pandemics: health care emergencies. In: Ursano RJ, Fullerton CS, Weisaeth L, et al, editors. Textbook of Disaster Psychiatry. Cambridge; Cambridge University Press. 2017 p.

4. Pfefferbaum B, North CS. Mental health and the Covid-19 pandemic. $N$ Engl J Med. 2020;383(6):510-512.

5. Brooks SK, Webster RK, Smith LE, et al. The psychological impact of quarantine and how to reduce it: rapid review of the evidence. Lancet. 2020;395(10227):912-920.

6. Lau JTF, Yang X, Pang E, et al. SARS-related perceptions in Hong Kong. Emerg Infect Dis. 2005;11(3):417-424.

7. Fernandez-Mendoza J, Vgontzas AN. Insomnia and its impact on physical and mental health. Curr Psychiatry Rep. 2013;15(12):418.

8. Kumar A, Goel H, Nadar SK. Short sleep duration and the risk of hypertension: snoozing away high blood pressure? J Hum Hypertens. 2019;33(3):174-176.

9. Morin CM, Jarrin DC. Epidemiology of insomnia: Prevalence, course, risk factors, and public health burden. Sleep Med Clin. 2013;8(3):P281297.

10. Ohayon MM. Epidemiology of insomnia: what we know and what we still need to learn. Sleep Med Rev. 2002;6(2):97-111. 
11. Kryger MH, Dement WC, Roth T. Principles and Practice of Sleep Medicine, 5th Edition. 2010.

12. Basta M, Chrousos GP, Vela-Bueno A, et al. Chronic insomnia and the stress system. Sleep Med Clin. 2007;2(2):279-291.

13. Shi L, Lu ZA, Que JY, et al. Prevalence of and risk factors associated with mental health symptoms among the general population in china during the coronavirus disease 2019 pandemic. JAMA Netw Open. 2020;3(7):e2014053.

14. Huang Y, Zhao N. Generalized anxiety disorder, depressive symptoms and sleep quality during COVID-19 outbreak in China: a web-based cross-sectional survey. Psychiatry Res. 2020;288:112954.

15. Guo J, Feng XL, Wang XH, et al. Coping with COVID-19: exposure to covid-19 and negative impact on livelihood predict elevated mental health problems in Chinese adults. Int $J$ Environ Res Public Health. 2020;17(11):3857.

16. Fu W, Wang C, Zou L, et al. Psychological health, sleep quality, and coping styles to stress facing the COVID-19 in Wuhan, China. Transl Psychiatry. 2020;10(1):225.

17. Voitsidis $P$, Gliatas I, Bairachtari V, et al. Insomnia during the COVID-19 pandemic in a Greek population. Psychiatry Res. 2020;289:113076.
18. Gualano MR, Lo Moro G, Voglino G, et al. Effects of COVID-19 lockdown on mental health and sleep disturbances in Italy. Int $J$ Environ Res Public Health. 2020;17(13):4779.

19. Kokou-Kpolou CK, Megalakaki O, Laimou D, et al. Insomnia during COVID-19 pandemic and lockdown: prevalence, severity, and associated risk factors in French population. Psychiatry Res. 2020;290:113128.

20. Pieh C, Budimir S, Probst T. The effect of age, gender, income, work, and physical activity on mental health during coronavirus disease (COVID-19) lockdown in Austria. J Psychosom Res. 2020;136:110186.

21. Yu BY-M, Yeung W-F, Lam JC-S, et al. Prevalence of sleep disturbances during COVID-19 outbreak in an urban Chinese population: a crosssectional study. Sleep Med. 2020;74:18-24.

22. Lin LY, Wang J, Ou-Yang XY, et al. The immediate impact of the 2019 novel coronavirus (COVID-19) outbreak on subjective sleep status. Sleep Med. 2020;S1389-S9457(20)30221-30225.

23. Li Y, Qin Q, Sun Q, et al. Insomnia and psychological reactions during the COVID-19 outbreak in China. J Clin Sleep Med. 2020;16(8):14171418 . 